\title{
Finite element simulation of slant cantilever beam and approximate equation formula of its resonance frequency
}

\author{
Manabu Aoyagi, Kenji Sato, and Yoshiro Tomikawa \\ Faculty of Engineering, Yamagata University, \\ 4-3-16, Jonan, Yonezawa, 992 Japan
}

(Received 21 June 1995)

\begin{abstract}
The first part of this paper deals with the finite element simulation of a slant cantilever beam, and the second part contains an approximate equation formula of its resonance frequency. We investigated such vibrational behaviors of a slant cantilever beam because we had to design a large-diameter torsional vibrator with many slant slits cut into its end-face for a rod-type ultrasonic motor. Also, we thought that the simulated and investigated results on the slant cantilever beam might be very useful in other applications, for example, vibrational sensors. In this paper, therefore, we present some finite element simulations of a slant cantilever beam and we investigate the results of its approximate equation formula, as academic research material.
\end{abstract}

Keywords: Slant cantilever beam, Finite element simulation, Approximate equation formula, Vibration mode pattern, Resonance frequency

PACS number: $43.40 . \mathrm{CW}$

\section{INTRODUCTION}

We have previously endeavored to develop several kinds of ultrasonic motors. ${ }^{1-3)}$ One of them is the rod-type ultrasonic motor. ${ }^{4}$ This ultrasonic motor has a large diameter and is designed for high torque. The ability of an ultrasonic motor depends on the vibrational characteristics of the stator vibrator. Therefore, we wanted to use a large-diameter torsional vibrator with many slant slits cut into its end-face, so that, in order to rotate the rotor, their flexural vibration was necessary. In this case, fundamental vibration-characteristics of the slant cantilever beam were important for good performance, and then we realised that vibration-characteristics of the slant cantilever beam, for example, its resonance frequencies and vibration modes, had to be simulated. From such practical engineering demands, we simulated the slant cantilever beam. The simulation was done by the finite element method (FEM) of the modified SAP IV Structual Analysis
Program. ${ }^{5)}$ From the simulated results, we discovered resonance frequencies and vibration modes of the slant cantilever beam. Moreover, we investigated an approximate equation formula for the flexural vibration resonance frequency of a slant cantilever beam.

This paper deals with the finite element simulation of the slant cantilever beam and the approximate equation formula of its resonance frequency. The simulated results for the cantilever beam will be very useful not only in designing a torsional stator vibrator for an ultrasonic motor, but also in other applications of the slant cantilever beam. Therefore, we present some simulated data of the slant cantilever beam as academic research material.

\section{FINITE ELEMENT SIMULATION OF A SLANT CANTILEVER BEAM}

\subsection{Slant Cantilever Beam}

The slant cantilever beam dealt with in this paper 
is shown in Fig. 1, together with a normal cantilever beam. The slant cantilever beam in Fig. 1(b) is the same as the normal cantilever beam in Fig. 1(a) when inclined horizontally. The detailed form of the slant cantilever beam is shown in Fig. 2. The main problem we wanted to consider is the flexural vibration characteristic in the $x-y$ horizontal plane; however, in the finite element simulation, some fundamental modes of flexural vibration in the $x-z$ vertical plane and longitudinal or torsional vibration are also considered.

\subsection{Finite Element Method (FEM)}

The slant cantilever beam shown in Fig. 2 was divided as shown in Fig. 3 for the FEM simulation. There are 385 nodal points and 240 divided elements. It is thought that these divisions in Fig. 3 might be enough for simulation of the first and second flexural vibrations, the first longitudinal vibration, the first torsional vibration. In the FEM simulation, it was supposed that the slant cantilever

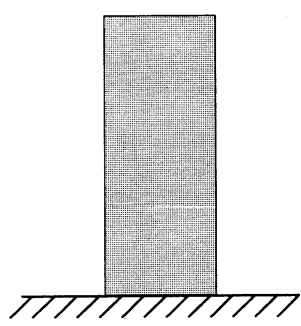

(a) Normal Cantilever Beam

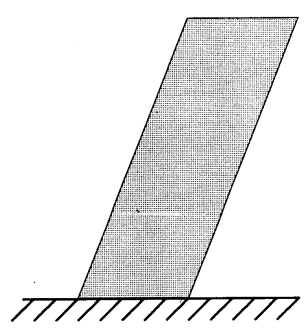

(b) Slant Cantilever Beam
Fig. 1 Normal cantilever beam and slant cantilever beam.

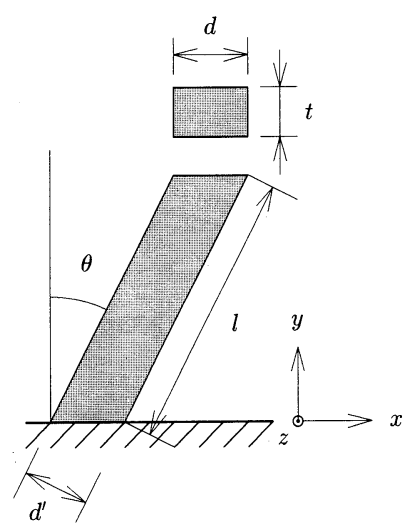

Fig. 2 Form of slant cantilever beam. beam was composed of piezoelectric ceramics, and for its material constants, the ones of the piezoelectric ceramics C-201 of Fuji Ceramics Co. Ltd. were used. The material constants of C-201 piezoelectric ceramics are presented in Table 1.

The FEM program used in the simulation is the SAP IV structural analysis program, which was modified to deal with piezoelectric vibration and is fundamentally applicable to anisotropic material and three-dimensional vibration problems. ${ }^{5)}$ The boundary condition for the FEM simulation was that nodal points of the support part were fixed and the other nodal points were free.

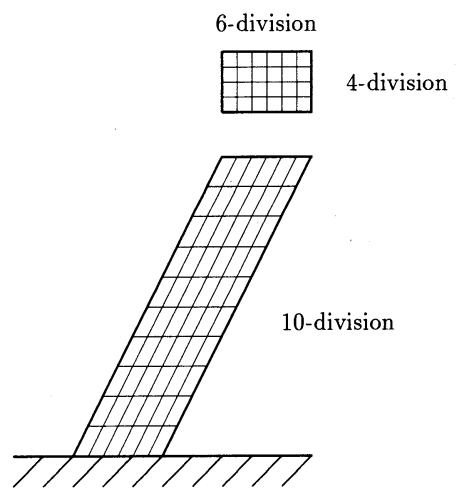

Fig. 3 Finite element divisions of slant cantilever beam.

Table 1 Material constants of piezoelectric ceramics C-201 (Fuji Ceramics Co. Ltd.).

\begin{tabular}{lllr}
\hline \multicolumn{1}{c}{ Property } & Symbol & Unit & \\
\hline Coupling factor & $k_{\mathrm{r}}$ & & 0.595 \\
& $k_{31}$ & & 0.346 \\
& $k_{33}$ & & 0.714 \\
& $k_{\mathrm{t}}$ & & 0.473 \\
& $k_{15}$ & & 0.735 \\
$C$ constant & $C_{11}^{\mathrm{E}}$ & $\mathrm{N} / \mathrm{m}^{2}$ & $14.9 \times 10^{10}$ \\
& $C_{12}^{\mathrm{E}}$ & $\mathrm{N} / \mathrm{m}^{2}$ & $8.7 \times 10^{10}$ \\
& $C_{13}^{\mathrm{E}}$ & $\mathrm{N} / \mathrm{m}^{2}$ & $9.1 \times 10^{10}$ \\
& $C_{33}^{\mathrm{E}}$ & $\mathrm{N} / \mathrm{m}^{2}$ & $13.2 \times 10^{10}$ \\
& $C_{44}^{\mathrm{E}}$ & $\mathrm{N} / \mathrm{m}^{2}$ & $2.5 \times 10^{10}$ \\
& $C_{66}^{\mathrm{E}}$ & $\mathrm{N} / \mathrm{m}^{2}$ & $3.1 \times 10^{10}$ \\
$e$ constant & $e_{31}$ & $\mathrm{C} / \mathrm{m}^{2}$ & -4.5 \\
& $e_{33}$ & $\mathrm{C} / \mathrm{m}^{2}$ & 17.5 \\
$\varepsilon$ constant & $e_{15}$ & $\mathrm{C} / \mathrm{m}^{2}$ & 15.2 \\
& $\varepsilon_{11}^{\mathrm{s}} / \varepsilon_{0}$ & & 904 \\
Poission's ratio & $\varepsilon_{33}^{\mathrm{s}} / \varepsilon_{0}$ & & 818 \\
Mechanical quality factor & $\sigma^{\mathrm{E}}$ & & 0.29 \\
Density & $Q_{\mathrm{m}}$ & & 889 \\
& $\rho$ & $\mathrm{kg} / \mathrm{m}^{3}$ & $7.82 \times 10^{3}$ \\
\hline
\end{tabular}




\subsection{Simulated Results ${ }^{6)}$}

In the simulation, we gave the simulated vibration modes simplified names, as shown in Table 2. Simulations of the resonance frequency $f_{\mathrm{r}}$ and mode patterns were done, for example, under such conditions that width $d$, thickness $t$, and slant angle $\theta$ of the cantilever beam were changeable, but the length $l$ is kept constant at $l=10 \mathrm{~mm}$. Simulated vibration mode patterns of the slant cantilever beam at the slant angle $\theta=30^{\circ}$ are shown in Figs. $4 \sim 9$, in comparison with the vibration modes of the normal cantilever beam. It was clear that, in the case of the slant cantilever beam, the vibration mode patterns were much influenced and modified; that is, the following results were obtained:

(1) The L1-mode was found to be modified to the H2-mode by inclining the normal cantilever beam ; that is, distinction between the L1- and $\mathrm{H} 2$-mode vibrations was not so easy, and it can be said that the L1-mode accompanied the

Table 2 Expression of vibration modes.

\begin{tabular}{lc}
\hline \multicolumn{1}{c}{ Vibration mode } & Simplified name \\
\hline First flexural vibration (horizontal direction) & H1-mode \\
Second flexural vibration (horizontal direction) & H2-mode \\
First flexural vibration (vertical direction) & V1-mode \\
Second flexural vibration (vertical direction) & V2-mode \\
First longitudinal vibration & L1-mode \\
First torsional vibration & T1-mode \\
\hline
\end{tabular}
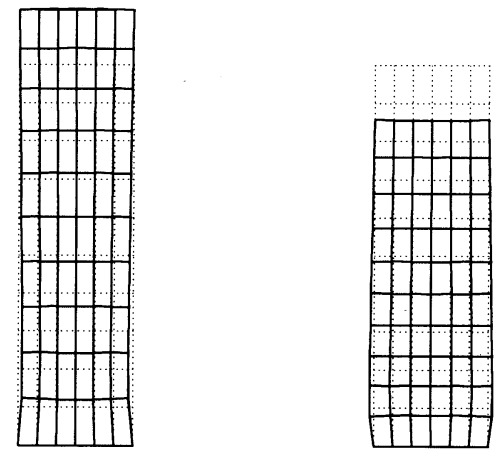

(a) Normal cantilever beam

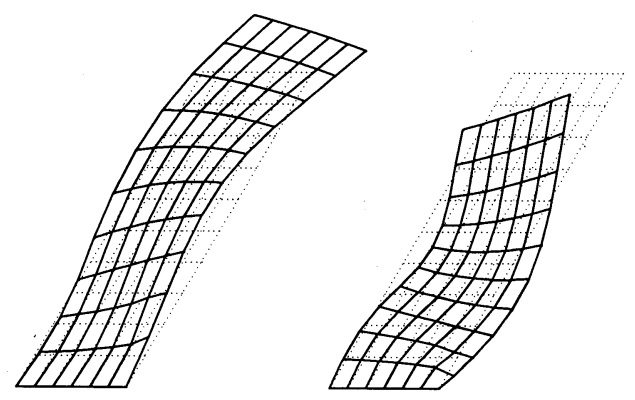

(b) Slant cantilever beam $\left(\theta=30^{\circ}\right)$

Fig. 4 Simulated vibration mode patterns of L1-mode.

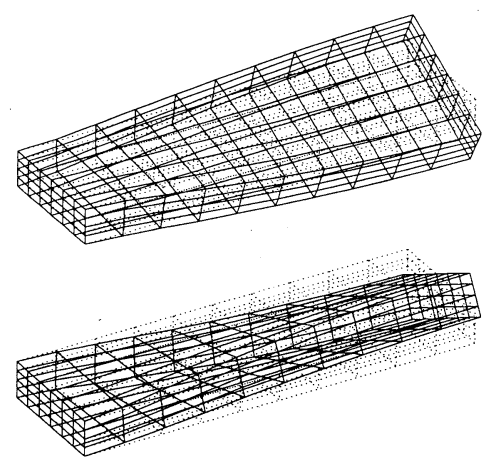

(a) Normal cantilever beam

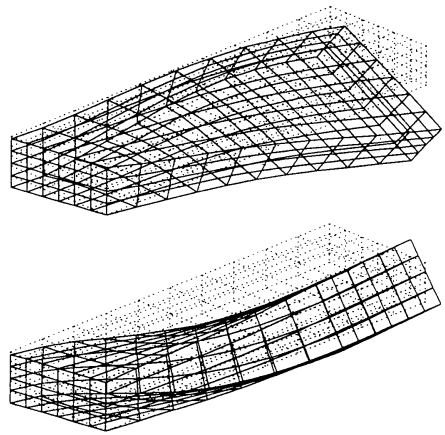

(b) Slant cantilever beam $\left(\theta=30^{\circ}\right)$

Fig. 5 Simulated vibration mode patterns of T1-mode. 

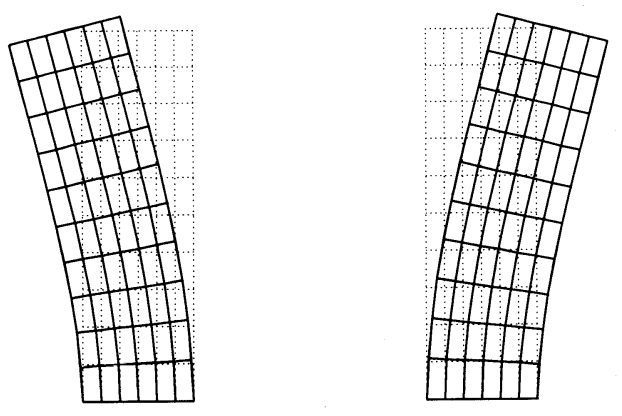

(a) Normal cantilever beam
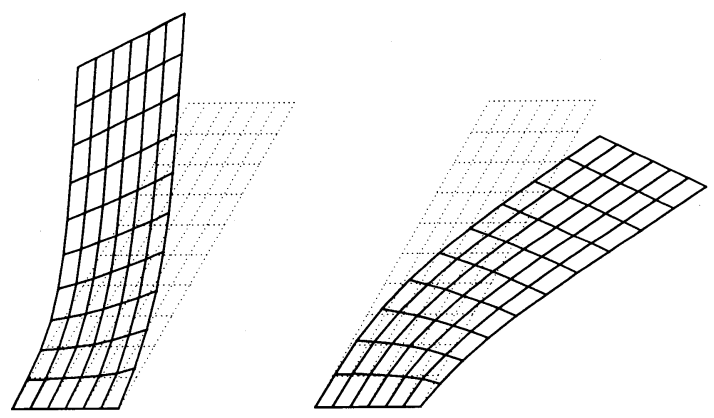

(b) Slant cantilever beam $\left(\theta=30^{\circ}\right)$

Fig. 6 Simulated vibration mode patterns of H1-mode.

H2-mode in the slant cantilever beam. Therefore, the top of the cantilever beam makes an elliptic motion. It is thought that this vibration mode could be applied to an actuator due to the elliptic motion.

(2) In the T1-mode, by inclining the normal cantilever beam, the symmetry of the form, which is regarded as the vibration axis of the torsional vibration, was lost. Therefore, an asymmetrical torsional vibration occurred in the cantilever beam. In other words, the component of the flexural vibration was largely contained in the displacements.

(3) H1-mode and H2-mode

In both the H1-mode and the H2-mode, displacement in the longitudinal-direction was found. Therefore, an elliptic motion occurred on the top of the slant cantilever beam in the same manner as in the L1-mode, but the direction of its rotation was different. Moreover, it was found that the resonance frequencies of the
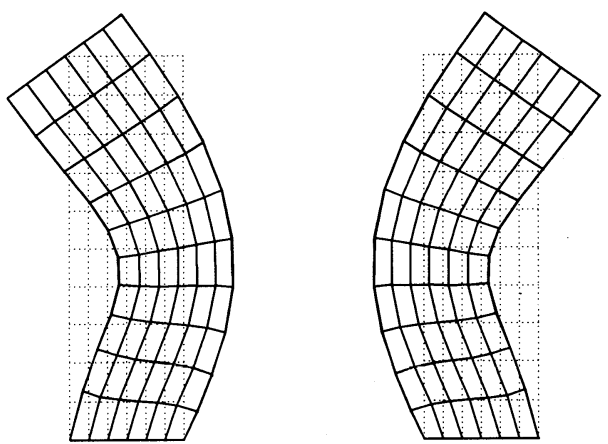

(a) Normal cantilever beam
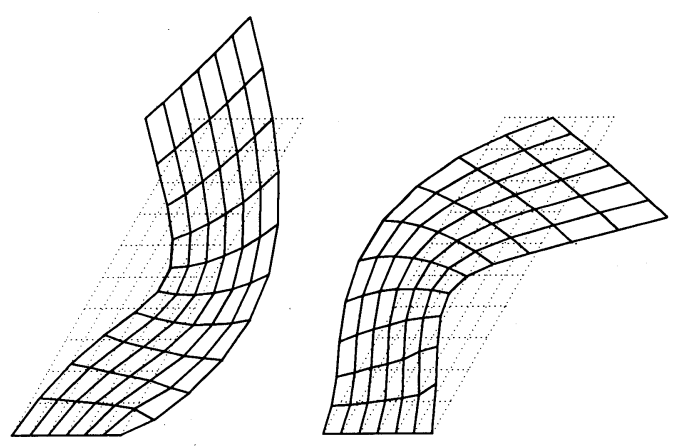

(b) Slant cantilever beam $\left(\theta=30^{\circ}\right)$

Fig. 7 Simulated vibration mode patterns of H2-mode.

H1- and H2-modes decreased with the slant angle. Therefore, we concluded that the simulated results of these vibration modes could be applied to the construction of an ultrasonic motor.

(4) V1-mode and V2-mode

Components of torsional vibration were also found in the displacement of both V1- and V2-modes. It was thought that slippage of the symmetry axis was caused by inclination. Moreover, by inclination, such simple and pure flexural vibration, as is found in the normal cantilever beam, was not found.

\section{APPROXIMATE EQUATION FORMULA OF FLEXURAL VIBRATION RESONANCE FREQUENCY}

3.1 Resonance Frequency of a Cantilever Beam The resonance frequency of a normal cantilever 


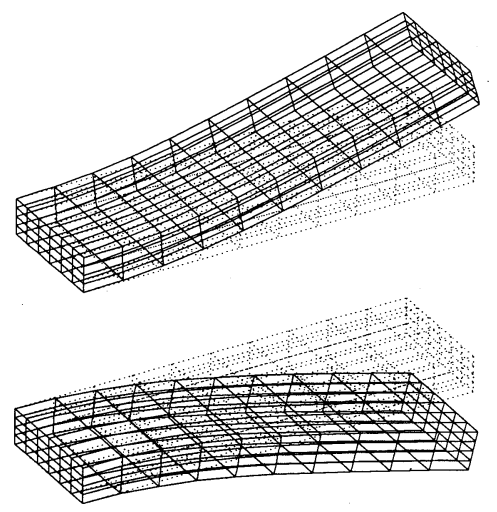

(a) Normal cantilever beam

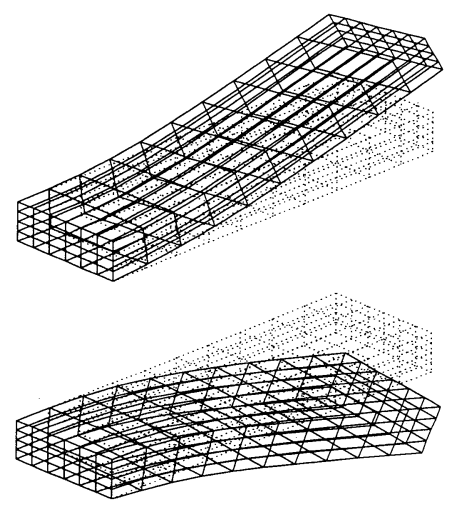

(b) Slant cantilever beam $\left(\theta=30^{\circ}\right)$

Fig. 8 Simulated vibration mode patterns of V1-mode.

beam flexurally vibrating, as shown in Fig. 1(a) and Fig. $2\left(\theta=0^{\circ}\right)$, can be expressed by the following equation

$$
\begin{aligned}
f_{n} & =\frac{\alpha_{n}^{2}}{2 \pi l^{2}} \sqrt{\frac{E I}{\rho A}} \\
& =\frac{\alpha_{n}^{2}}{2 \pi l^{2}} \sqrt{\frac{E}{\rho}} \frac{d}{\sqrt{12}},
\end{aligned}
$$

where each parameter has the following meaning;

$n$ : resonance mode number

$\alpha_{n}$ : frequency constant, $\alpha_{1}=1.875$

$E$ : Young's modulus

$I$ : second moment of area

$\rho$ : material density

$A$ : cross-sectional area $(d \times t)$

In the case of the slant cantilever beam, if an equation formula such as Eq. (1) is obtained, it can be very useful for applying the slant cantilever beam to an actuator or a sensor device, and so on. There-

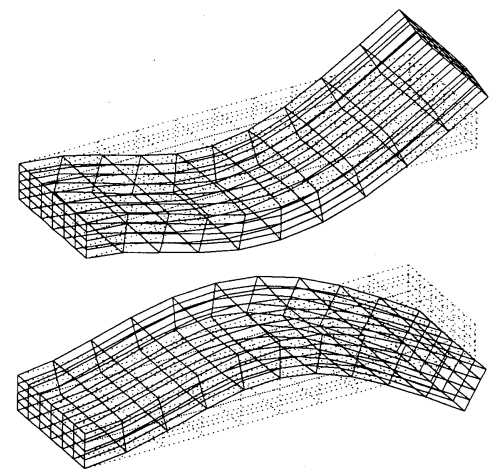

(a) Noraml cantilever beam
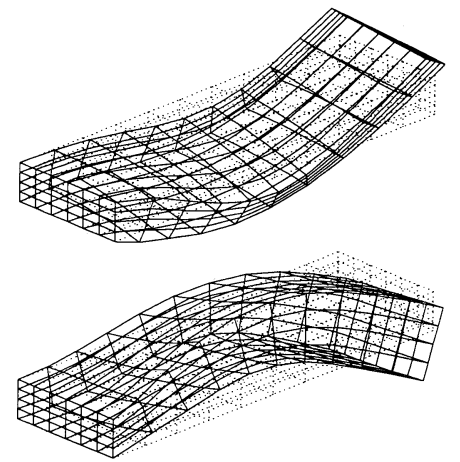

(b) Slant cantilever beam $\left(\theta=30^{\circ}\right)$

Fig. 9 Simulated vibration mode patterns of V2-mode.

fore, we determined the approximate equation formula of resonance frequency on the slant cantilever beam flexurally vibrating in the $x-y$ plane in Fig. 2 .

3.2 Approximate Equation Formula of Slant Cantilever Beam Resonance Frequency

Before obtaining the approximate equation formula of resonance frequency, we determined Young's modulus $E$ in Eq. (1) by following the considerations in the FEM simulation, because the piezoelectric material C-201 used in the simulation is an anisotropic elastic body : that is, in the case of the normal cantilever beam, Eq. (2) is obtained from Eq. (1) as :

$$
E=f_{n}^{2}\left(\frac{2 \pi l^{2}}{\alpha_{n}^{2}}\right)^{2} \frac{\rho A}{I} .
$$

Therefore, substituting the simulated 1st flexural resonance frequency in Eq. (2), Young's modulus $E$ was obtained with precision as shown in Table 3 
and determined as $E=9.356 \times 10^{11}\left[\mathrm{gcm}^{-1} \mathrm{~s}^{-2}\right]$. Therefore, using Eq. (1) and applying Young's modulus $E$, as determined above, the 1st flexural resonance frequency was calculated as shown in Table 4, when the length $l$ and form-ratio $t / d$ of a normal cantilever were $10 \mathrm{~mm}$ and 0.1 , respectively. Consequently, it is estimated that the reliability of the FEM simulation was improved by applying the Young's modulus $E$, as determined above.

Using these results, we derived the approximate equation formula which could be applied to the flexural resonance frequency of a slant cantilever beam, under such a condition that the cantilever beam was made to slant with its constant length $l$ in Fig. 2. Therefore, the width $d^{\prime}$ of the slant cantilever beam is related to the width $d$ of a normal cantilever beam as follows :

$$
d^{\prime}=d \cos \theta
$$

Substituting Eq.(3) into Eq. (1), Eq. (1) is modified to Eq. (4).

$$
\begin{aligned}
f_{\mathrm{H} n}{ }^{\prime \prime} & =\frac{\alpha_{n}{ }^{2}}{2 \pi l^{2}} \sqrt{\frac{E}{\rho}} \frac{d^{\prime}}{\sqrt{12}} \\
& =\frac{\alpha_{n}{ }^{2}}{2 \pi l^{2}} \sqrt{\frac{E}{\rho}} \frac{d}{\sqrt{12}} \cos \theta
\end{aligned}
$$

If the width $d$ of the cantilever beam becomes width $d^{\prime}$ of the slant cantilever beam, the resonance frequency constant $\alpha_{n}$ will be changed to a function of $d^{\prime}$, because it depends on $d^{\prime} / l$. The values of $\alpha_{n}^{\prime}$

Table 3 Young's modulus obtained by resonance frequencies.

\begin{tabular}{ccc}
\hline$d / l$ & $\begin{array}{c}\text { Resonance frequency } \\
f_{\mathrm{H} 1}[\mathrm{kHz}]\end{array}$ & $\begin{array}{c}\text { Young's modulus } \\
E\left[\mathrm{~N} / \mathrm{m}^{2}\right]\end{array}$ \\
\hline 0.1 & 5.544 & $9.356 \times 10^{10}$ \\
0.3 & 15.689 & $9.335 \times 10^{10}$ \\
0.5 & 23.760 & $9.316 \times 10^{10}$ \\
0.7 & 29.750 & $9.308 \times 10^{10}$ \\
1.0 & 35.839 & $9.318 \times 10^{10}$ \\
\hline
\end{tabular}

were obtained on Ref.7). Therefore, Eq. (4) becomes Eq. (5).

$$
\begin{gathered}
f_{\mathrm{H} n}{ }^{\prime}=\frac{\alpha_{n}{ }^{2}}{2 \pi l^{2}} \sqrt{\frac{E}{\rho}} \frac{d}{\sqrt{12}} \cos \theta \\
\alpha_{n}^{\prime}: \text { frequency constant } \\
\text { (slant cantilever beam) }
\end{gathered}
$$

The $f_{\mathrm{H} n}{ }^{\prime}$ calculated from Eq. (5) and the simulated resonance frequency from the FEM simulation are shown in Fig. 10. From these results, it was found that the resonance frequency was changed by the dimensional ratio $d / l$; however, the theoretical resonance frequency from Eq. (5) and the simulated frequency were almost equal to each other when $d / l=0.1$, and then as the ratio $d / l$ increases from 0.1 to 1.0 , the difference between them increased. Therefore, we revised the theoretical equation of $f_{\mathrm{H} n}$ to Eq. (6) in consideration of the influence of $d / l$; that is,

$$
\begin{aligned}
f_{\mathrm{H} n_{-} \text {approximate }}= & \left\{\frac{d}{l} f_{n}\right\}+\left\{\left(1-\frac{d}{l}\right) f_{\mathrm{H} n^{\prime}}\right\} \\
= & \left\{\alpha_{n}{ }^{2} \frac{d}{l}+\alpha_{n}{ }^{2}\left(1-\frac{d}{l}\right) \cos \theta\right\} \\
& \cdot \frac{d}{2 \sqrt{12} \pi l^{2}} \sqrt{\frac{E}{\rho}}
\end{aligned}
$$

Equation (6) is called an approximate equation formula (abbreviated to AEF hereafter) for obtaining the resonance frequency of the slant cantilever beam.

\subsection{Comparison between Resonance Frequencies} Calculated by AEF and Simulated by FEM

Both resonance frequencies calculated by the AEF of Eq. (6) and simulated by FEM are shown in Figs. $11(\mathrm{a}) \sim(\mathrm{e}),{ }^{8)}$ where we present the values of $f_{\mathrm{H} 1}{ }^{\prime}$ calculated by Eq. (5), too. From these results, it is proven that the flexural resonance frequency $\left(f_{\mathrm{H} 1}\right)$ can be approximately calculated, with enough exactness, in regard to the H1-mode. However the AEF of Eq. (6) might produce a noticeable error when calculating the H2-mode flexural resonance fre-

Table 4 Flexural resonance frequencies obtained by FEM and by theoretical calculation.

\begin{tabular}{cccc}
$\begin{array}{c}\text { Form ratio } \\
d / l\end{array}$ & $\begin{array}{c}\text { Resonance frequency } \\
\text { (for simulation) } \\
f_{\mathrm{H} 1}[\mathrm{kHz}]\end{array}$ & $\begin{array}{c}\text { Resonance frequency } \\
\text { (for theoretical calculation) } \\
f_{1}[\mathrm{kHz}]\end{array}$ & $\begin{array}{c}\text { Error } \\
{[\%]}\end{array}$ \\
0.1 & 5.544 & 5.544 & \pm 0.00 \\
0.3 & 15.689 & 15.707 & -0.11 \\
0.5 & 23.760 & 23.811 & -0.21 \\
0.7 & 29.750 & 29.826 & -0.25 \\
1.0 & 35.839 & 35.912 & -0.20 \\
\hline
\end{tabular}




\section{AOYAGI et al.: FEM SIMULATION OF SLANT CANTILEVER BEAM}

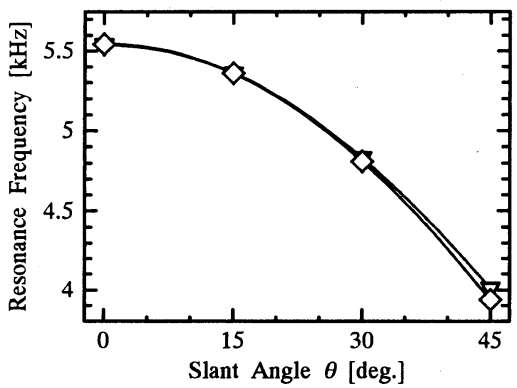

(a) $d / l=0.1$

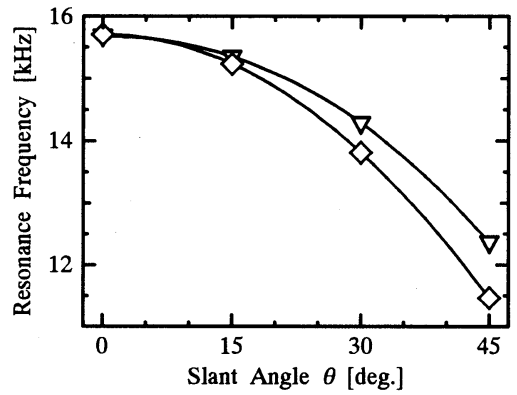

(b) $d / l=0.3$

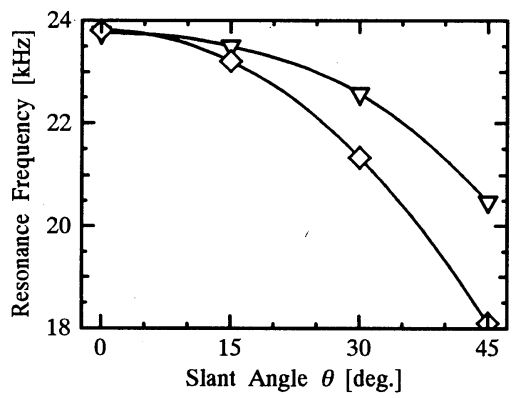

(c) $d / l=0.5$

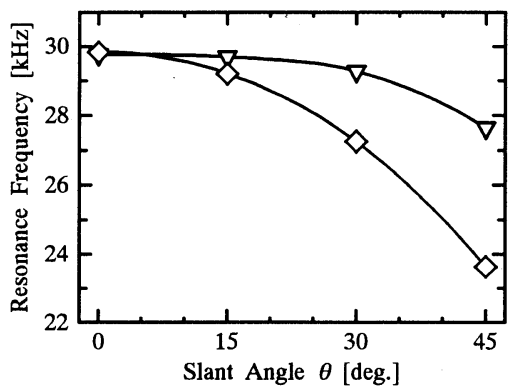

(d) $d / l=0.7$

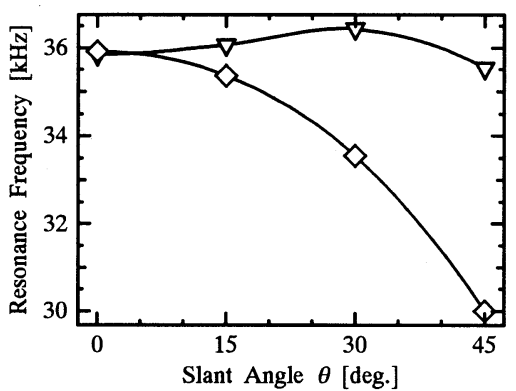

(e) $d / l=1.0$

Fig. 10 Simulated and calculated flexural frequencies.

quency, because the H2-mode combines considerably with the L1-mode.

\section{SUMMARY}

Resonance mode patterns and resonance frequencies of the slant cantilever beam were simulated by the finite element method. Moreover, the approximate equation formula to calculate the resonance frequency of the H1-mode was investigated. These results are summarized as follows:

(1) It became clear that the vibration mode patterns are greatly influenced by slanting.

(2) The bending vibration component of displacement is also generated in the L1-mode, and an elliptic motion is formed on the top of the slant cantilever beam.

(3) The bending vibration components are generated in the T1-mode, too.

(4) In the H1- and H2-modes also, the longitudinal displacements generate an elliptic motion.

(5) The torsional displacements are generated in the V1-mode and the V2-mode, but in the torsional vibration mode, the displacements on the top of the slant cantilever beam are influenced little.

(6) We obtained the approximate equation formula to calculate the H1-mode flexural resonance frequency of the slant cantilever beam. 


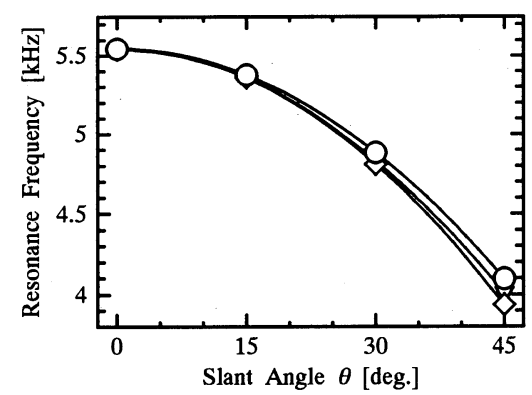

(a) $d / l=0.1$

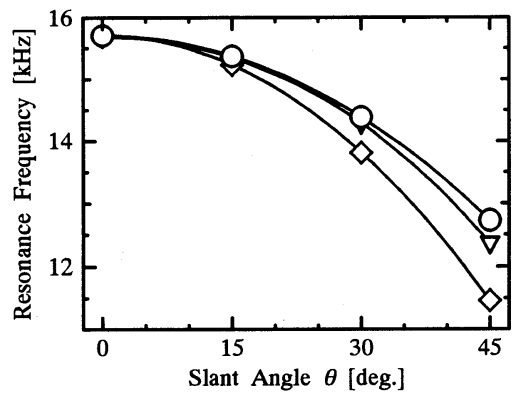

(b) $d / l=0.3$

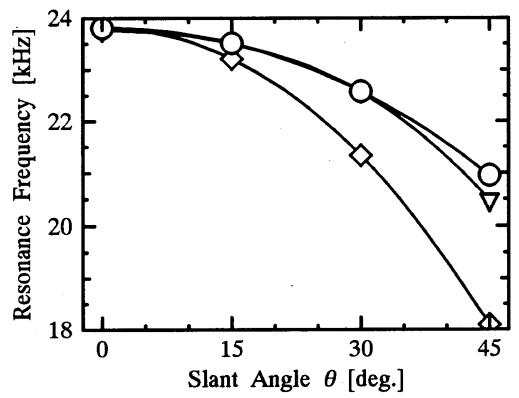

(c) $d / l=0.5$

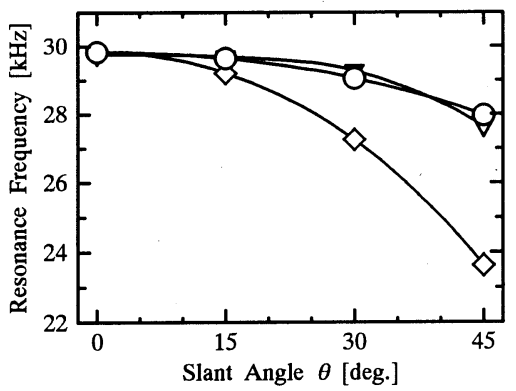

(d) $d / l=0.7$

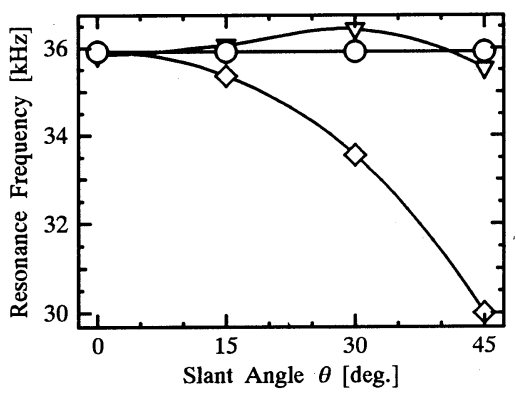

(e) $d / l=1.0$

Fig. 11 Flexural resonance frequencies by FEM simulation and by calculation with AEF.

(7) In the estimation of resonance frequencies, Young's modulus was determined so that the results simulated by FEM were made equal to the calculated results.

As mentioned above, we clarified the vibrational behavior of the slant cantilever beam. The academic research material dealt with in this paper might be applicable not only to the construction of ultrasonic motors using the slant-slit torsional vibrator, but also to other demands.

\section{REFERENCES}

1) S. Ueha and Y. Tomikawa, Ultrasonic Motor - Theory and Applications - (Oxford Science Publi-
cation/Clarendon Press, Oxford, 1993).

2) Y. Tomikawa, "Piezoelectric ultrasonic motors," Actuators Reported in 1992 and 1993 by Research Group of Yamagata University and Tohoku Institute of Technology, 1993 JITAS 37th Usual Conference on Actuators and Transducers (1993).

3) Y. Tomikawa, T. Takano, C. Kusakabe, M. Aoyagi, and S. Hirose, "Development of ultrasonic motors," 9th Smart Actuator Symposium at International Conference on Actuator and Transducers (ICAT), The Pennsylvania State University (1994).

4) K. Sato, M. Aoyagi, T. Ogasawara, and Y. Tomikawa, "Ultrasonic motor using a large-diameter torsional vibrator with slant slits," Jpn. J. Appl. Phys. 34, 2707-2710 (1995).

5) Y. Tomikawa, H. Miura, and S. B. Dong, "Analysis 


\section{AOYAGI et al.: FEM SIMULATION OF SLANT CANTILEVER BEAM}

of electrical equivalent circuit elements of piezotuning forks by the finite element method," IEEE Trans. Sonics Ultrason. SU-25, 206-212 (1978).

6) K. Sato, M. Aoyagi, and Y. Tomikawa, "F. E. M. simulation of slant cantilever beam vibration mode," Proc. 1994 IEICE Fall Conf., No. A-219, 220 (1994).

7) S. Sugawara, "Fukugōkikaishindōkei no Tōkakairomōkaiseki to sono Filter eno Ōyō," A Thesis for D.E. Degree, Tohoku University, Japan, 13, 18-20 (1983) (in Japanese).

8) K. Sato, M. Aoyagi, and Y. Tomikawa, "Flexural resonance frequencies of a slant cantilever beam," Proc. 1994 IEICE Fall Conf., No. A-218, 219 (1994).

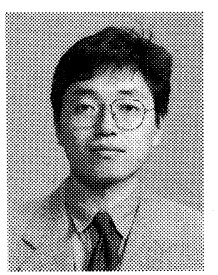

Manabu Aoyagi was born in Yamagata Prefecture, Japan, on September 6,1966 . He received the B.E. and the M.E. degrees in electrical engineering from Yamagata University, Yonezawa, Japan, in 1989 and 1991, respectively. He is now a research associate at Yamagata University. He received the Young Engineer Award from the Institute of Electronics, Information and Communication Engineers of Japan in 1994. His research interests include electromechanical transducers and ultrasonic motors. Mr. Aoyagi is a member of the Acoustical Society of Japan and the Institute of Electronics, Information and Communication Engineers of Japan.

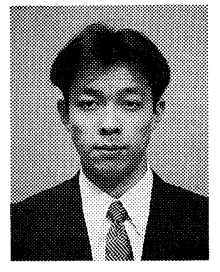

Kenji Sato was born in Miyagi Prefecture, Japan, on August 8, 1970. He received the B.E. degree from Ishinomaki Senshu University, Ishinomaki, Japan, and M.E. degree in electrical engineering from Yamagata University, Yonezawa, Japan, in 1993 and 1995, respectively. Since April 1995, he has been in Tokyo Communication Equipment Co., Ltd. Mr. Sato is a member of the Institute of Electronics, Information and Communication Engineers of Japan.

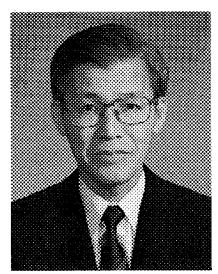

Yoshiro Tomikawa was born in Saitama, Japan, on October 12, 1939. He received the B.E. degree in electrical engineering in 1962 from Yamagata University, Yonezawa, Japan, and the Ph.D. degree in 1974 from the University of Tokyo, Tokyo, Japan. From April 1962 to December 1962, he worked in Hitachi Co. Ltd., Totsuka, Yokohama, Japan. Since January 1963, he has been with Yamagata University, where he is a Professor of Electrical Engineering. From April 1969 to March 1970, he was a Researcher at the Institute of Industrial Science, University of Tokyo, and from September 1975 to October 1976, he was a Visiting Scholar at the University of California, Los Angeles, CA. His research interest includes electromechanical resonators and ultrasound applications. Dr. Tomikawa is a member of the Institute of Electronics, Information and Communication Engineers of Japan, the Institute of Electrical Engineering Japan and the Acoustical Society of Japan. 